\title{
HIERARCHICAL MULTI-ATTRIBUTE DECISION SUPPORT APPROACH TO VIRTUAL ORGANIZATION CREATION
}

Toni Jarimo (1), Peter Ljubič (2), Iiro Salkari (1), Marko Bohanec (2), Nada Lavrač (2, 3), Martin Žnidaršič (2), Stefan Bollhalter (4), Jiri Hodik (5)

(1)VTT Technical Research Centre of Finland, toni.jarimo@vtt.fi, iiro.salkari@vtt.fi (2)JožefStefanInstitute, Ljubljana, Slovenia, peter.ljubic@ijs.si,marko.bohanec@ijs.si, nada.lavrac@ijs.si,martin.znidarsic@ijs.si

(3) Nova Gorica Polytechnic, Nova Gorica, Slovenia

(4)Virtuelle Fabrik AG, Arbon, Switzerland, stefan.bollhalter@virtuelle-fabrik.com (5) Czech Technical University, Prague, Czech Republic, hodik@labe.felk.cvut.cz

\begin{abstract}
The process of virtual organization (VO) creation can be seen as the selection of a set of organizations from a larger set, called a VO breeding environment (VBE). Often a VBE provides several possible VO configurations whose efficiency differs. The ultimate task is to evaluate all possible configurations in order to select the optimal VO configuration. The contribution of this paper is twofold. First, we formalize the VO creation process as a multi-attribute decision-making problem. Second, we suggest a hierarchy of attributes for computer-supported VO creation. Finally, we illustrate our approach using a subset of criteria, through a case of an existing $V B E$, the Virtuelle Fabrik $A G$.
\end{abstract}

\section{INTRODUCTION}

The process of creating Virtual Organizations (VO) has recently attracted the attention of researchers from various fields. One approach is that of CamarinhaMatos and Afsarmanesh (2003), who suggest that a pool of proactively cooperating organizations, called a Virtual Organization Breeding Environment (VBE), would be an efficient platform for creating VOs. Their idea is that a VBE provides a common structure for repeated VO creation, hence decreasing the costs related to ad hoc VO creation. Such a VBE structure includes, for instance, a common infrastructure, contracting templates and decision-support tools for agile VO creation.

This paper builds on the VBE context, focusing on supporting the selection of a good VO configuration from among the members of a VBE. Since the notion of VBE is relatively new, VO creation in a VBE is not yet well understood. Therefore, this paper proposes a general formalization of the problem, which can serve as a basis for future tool development.

The need for VO selection arises when the VBE, or its broker who is a marketer of the VBE (e.g. Katzy and Dissel, 2001), identifies a value creation opportunity ${ }^{i}$ 
and is willing to create a VO for performing the job. Like many other decisionmaking situations, VO selection necessitates the definition of case-specific selection criteria, some of which are likely to be conflicting. Common examples of conflicting criteria are the usual project measures, i.e. cost, time and quality.

Multi-criteria decision-analysis (MCDA) is a well developed mathematical theory providing methods to cope with conflicting objectives (see e.g. Keeney and Raiffa, 1976). Therefore we suggest MCDA as one possible approach to support decisions related to VO creation. In this paper, we restrict ourselves to the case of a single decision-maker, whose ultimate task is to evaluate several alternatives, i.e. feasible VO configurations, with regard to multiple criteria. Naturally, the evaluation of alternatives leads to their ranking, which supports the selection of the most suitable one. Often, the decision criteria are structured in an attribute hierarchy, which divides the general criteria into subcriteria and sub-subcriteria etc.

The remainder of this paper is structured as follows. Section 2 formalizes VO creation as a multi-attribute decision-making problem. Section 3 suggests an attribute hierarchy for the problem and Section 4 illustrates the model through a real-life example. Section 5 concludes and suggests topics for future research.

\section{A MULTI-CRITERIA MODEL FOR VO CREATION}

\subsection{Two Phases of VO Creation}

We consider VO creation as a two-step process. Phase 1 defines the set of all possible VO configurations that are capable of performing the value creation opportunity addressed. Specifically we say that a VO is capable of performing a task if it has the necessary competencies and resources available to the extent the task requires. We denote the set of capable VO configurations as the feasible set. We assume that a broker has made a work breakdown structure of the value creation opportunity, including competency and resource needs. This naturally has consequences for the feasible set, hence emphasizing the role of the broker.

Phase 2 attaches a value to each VO configuration in the feasible set, thus defining a preference order of feasible VO configurations. The value of a VO configuration is an aggregation of its scores on given criteria. For instance, the set of criteria could be \{skills, efficiency, network relations\}, which can be further developed into a attribute hierarchy. We discuss the attribute hierarchy in more detail in Section 3.

\subsection{Mathematical Modeling}

Following the notation of Liesiö et al. (2005), let $X$ denote the set of organizations, i.e. the VBE. All possible VO configurations from VBE $X$ is the power set $P=2^{X}$. We denote the feasible set by $P_{f},\left(P_{f} \subset P\right)$, and its elements, i.e. the capable VO configurations, by $\left\{p^{1}, \ldots, p^{m}\right\}$.

We let $n$ be the number of criteria against which the elements of $P_{f}$ are evaluated. For each $p^{j} \in P_{f}$ there exists a score vector $\mathbf{v}^{j}=\left[\begin{array}{lll}v_{1}^{j} & \ldots & v_{n}^{j}\end{array}\right]$, whose element $v_{i}^{j}$ denotes the score of configuration $p^{j}$ corresponding to the $i$-th criterion. The different criteria 
are weighted with weights $w_{i}>0$. Weights are normalized so that $w_{1}+\ldots+w_{n}=1$. The value of configuration $p^{j}$ is given by the additive value function $\nu^{j}=\Sigma_{i=1 \ldots n} w_{i} v_{i}^{j}$.

We divide the criteria into two categories: 1) Node Attributes and 2) Network Preparedness Attributes. The difference between these two groups is that Node Attributes are related to a single VBE member (node), whereas Network Preparedness Attributes reflect the overall (decrease in) efficiency of a VO configuration, due to difficulties that multilateral working induces. Hence a VO configuration consisting of a single VBE member has the highest possible score on all Network Preparedness Attributes. Section 3 provides an example of such a criteria hierarchy.

The requirement for existence of an additive value function is that the attributes are mutually preferentially independent. To clarify this concept, we first give an example of preferential independence: Say the quality level $p$ ' that a VO provides is deemed better than quality level $p$ ', at cost level $z$ '. If this holds for any cost level $z$, then quality is preferentially independent of costs. Furthermore, if every subset $Y$ of these attributes is preferentially independent of any other subset $Z$, then the attributes are mutually preferentially independent. (Keeney and Raiffa, 1976)

In some cases, the feasible set $P_{f}$ may be very large and the evaluation of all feasible configurations becomes practically impossible. Therefore we need approaches that efficiently distinguish good alternatives from $P_{r}$. One way to diminish the number of alternatives is to identify dominated VO configurations, i.e. configurations which are worse than another configuration on every criterion (Keeney and Raiffa, 1976). For instance, Liesiö et al. (2005) have developed "Robust Portfolio Modeling" for identifying all non-dominated options from among a large set of alternatives.

\section{ATTRIBUTE HIERARCHY}

This section proposes an attribute hierarchy, which illustrates the division of the common problem into 1) Node Attributes and 2) Network Preparedness Attributes (Figure 1). In addition, it gives an insight into the two-phase process of firstly defining the feasible set and secondly attaching value to the configurations.

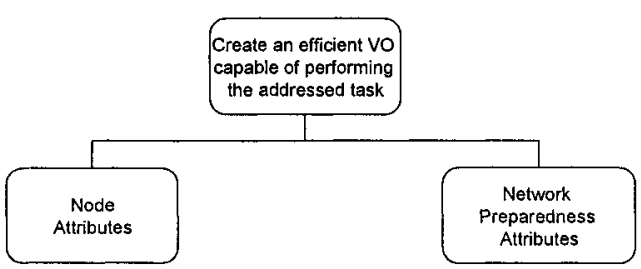

Figure 1 - Common Problem Divided into Two Attribute Categories

As discussed in Section 2, if a node has the required expertise for conducting some task defined in the work breakdown structure, the node is capable of the task. If the node has the required expertise, it is expected that the node has the necessary competencies, resources and availability for the task. We refer to these attributes as feasibility attributes. Reviewing the feasibility attributes reveals the feasible set, i.e. 
phase 1 of the VO creation process. Figure 2 presents the Node Attribute hierarchy, in which the feasibility attributes are marked with an asterisk $\left(^{*}\right)$.

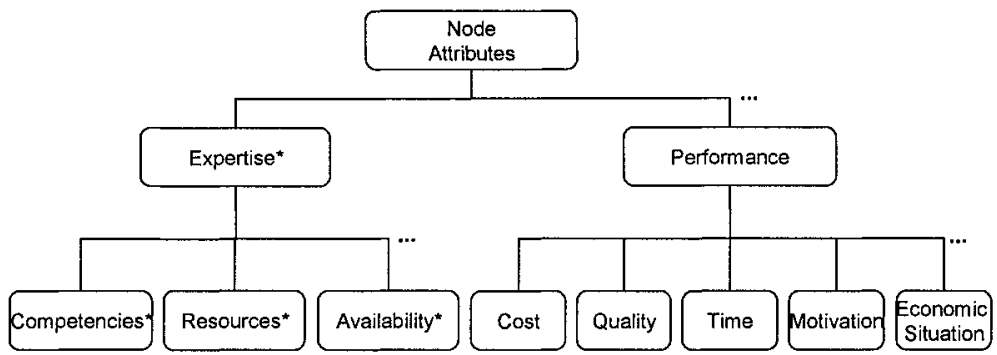

Figure 2 - Node attribute hierarchy, feasibility attributes marked with asterisk (*)

Phase 2 of the VO creation process attaches values to the different VO configurations of the feasible set. This takes place by assessing node performance individually and network preparedness as a whole. The performance of a single node is a node attribute and the related hierarchy is depicted in Figure 2. We propose that for instance cost, quality, time, motivation and economic situation effect the performance of a job the node is capable of.

The network preparedness attributes reflect the difficulties of multilateral working (Figure 3). They indicate the performance of a capable VO configuration. We propose that the network preparedness attributes can be divided into e.g. business characteristics, social characteristics and miscellaneous attributes. This division is not clear at every point nor is it exhaustive, but it illustrates our idea of network preparedness. Potential other attributes are identified by e.g. Lin and Chen (2004). Network preparedness attributes are issues that may make collaboration more difficult.

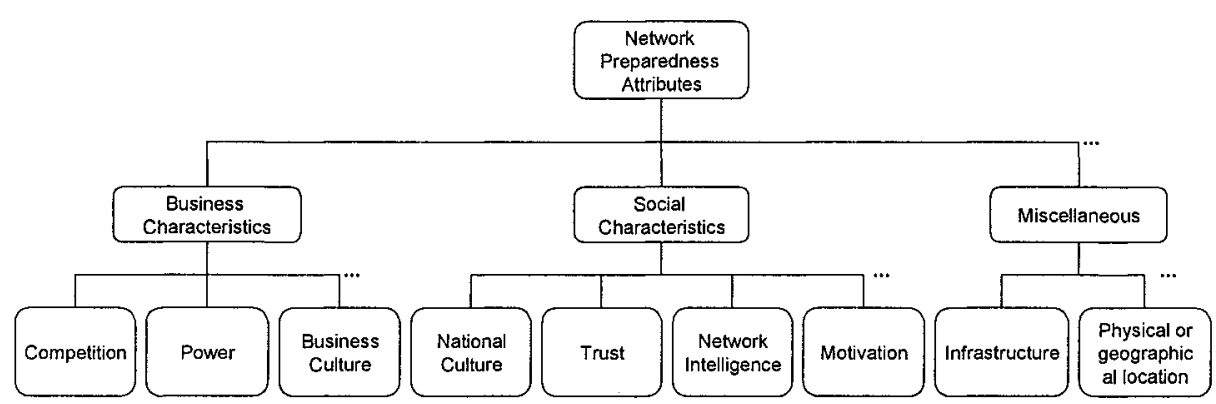

Figure 3-Network preparedness attributes

Business characteristics consist of competition, power and business culture. If companies compete for the same customers, it may be hard for them to collaborate, or even competition (or antitrust) law may prevent some companies from collaborating. Depending on the objective of the VO that is being created, the power difference of the companies may play a role: it may be desired, equal or restrictive from collaboration viewpoint. Companies that have interoperable infrastructure are in better situation when collaborating than companies with dissimilar or noninteroperable infrastructure. By network intelligence we mean accumulated 
experiences from past collaboration. Similarly, the rest of the attributes reflect the challenge of multilateral collaboration.

There is no general order of preference for attribute values. For instance, in the case of power, in a subcontracting type of collaboration, the power difference may even be desired by the principal company, whereas in joint R\&D efforts, the power difference may be considered as a drawback. Therefore, the decision-maker needs to elicit case-specific preference weights for attributes.

Weight elicitation methods for numerical MCDA models are numerous and well developed, such as WSM, ELECTRE, TOPSIS (Triantaphyllou, 2000), SMART and SMARTER (Edwards and Barron, 1994), and similar. A well-known method in this field is the Analytic Hierarchy Process (AHP) (Saaty, 1993), in which weights are elicited by pairwise comparison of attributes. AHP is interesting for its ability to estimate the consistency of assessment.

In addition, weight elicitation may require the use of qualitative variables as a complement to more typical quantitative ones. Qualitative variables are often denoted by words like "low", "appropriate" and "acceptable". The qualitative attributes are evaluated to suit decision-making problems that are less structured and formalized (Bohanec and Rajkovič, 1999). For qualitative (symbolic) multi-attribute models, a suitable method is implemented in the system called DEXi (Bohanec, 2003). The method approximates numerical weights from qualitatively assessed decision rules (Bohanec and Zupan, 2004). Regarding the decision support system, this implies that the system should be capable of analyzing both numerical and qualitative variables.

\section{AN ILLUSTRATIVE EXAMPLE CASE}

In this example case we tried to construct a rule-based hierarchical model using functional decomposition, which is focused on discovering novel concepts in the data. To build such a structure we used a hierarchy induction tool HINT (Zupan et al., 1999) from a data mining suite Orange (Demsar and Zupan, 2004). Its purpose is to decompose a complex function into a hierarchy of simpler ones. The result of such decomposition is a concept hierarchy with a number of tables that help to provide an extensional definition of concepts.

We provide an illustrative example using data of the Virtuelle Fabrik (VF) industry cluster in the area of mechanical engineering. The key question we would like to answer is the following: Can we not only predict, but also understand how a company's attributes influence its cooperation with other companies?

The data consists of two tables shown in Table 1. The first table describes a sample of 62 partners collaborating in VF and their attributes. The attributes are the following: activity, punctuality, reliability, motivation, love of risks and economic situation. All attributes can take values from 1 (very bad) to 6 (very good). The second table represents a cooperation matrix showing cooperation between companies. Values vary from 0 (no cooperation) to 3 (intense cooperation). Since input to the HINT functional decomposition should be in one table, we prepared the data in the following fashion. In the new table there is one row per each cooperative action of companies. Consequently, each company may appear in more than one row. Each row also holds information about the intensity of such cooperation, as 
shown in the lower table of Table 1. For simplicity we discretized companies' attribute values so that numbers 1 and 2 were changed to nominal value $l o w, 3$ and 4 to mid, and 5 and 6 to high. The rows with missing values were skipped (e.g. company 1 in table 1) and the attribute reliability with only one value (6) was removed. The resulting input table for HINT consists of 58 rows.

Table 1 - Information about companies (upper left-hand side table) and their cooperation (upper right-hand side table). For simplicity, zeros are omitted in the cooperation matrix. Below is the HINT input table, where shadowed rows represent the cooperation of company 4 (the shadowed row of the upper left-hand side table).

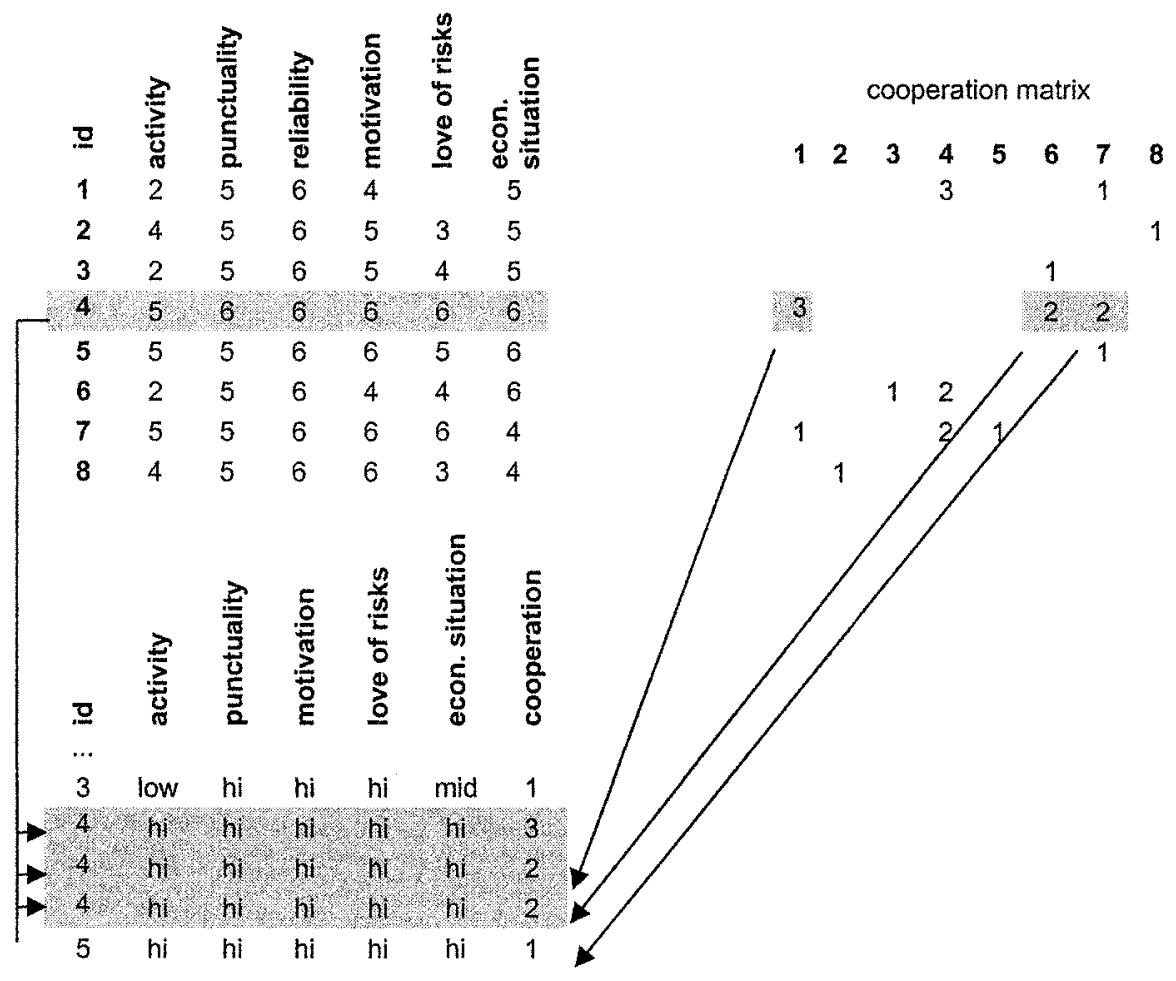

A result of HINT is a rule-based model for a hierarchy of concepts, shown in Figure 4 (note that due to space restrictions, inference rules are not listed). In the hierarchical structure, three new concepts are introduced (shown as ellipses in Figure 4), the numbers in brackets denote the number of different values for each new concept. What is the benefit of such a structure? We can use it as a classifier to estimate a new company's cooperation. However, a much greater value of this model lies in its transparency and the capability of using various analysis methods that work on multi-attribute decision-models (e.g. what-if). Such an automatically obtained model can serve as the first approximation of a model and as a starting point for an improvement in collaboration with a domain expert. An expert should 
recognize and label concepts according to his background knowledge, delete senseless concepts and possibly improve the data gathering for another iteration of concept formation.

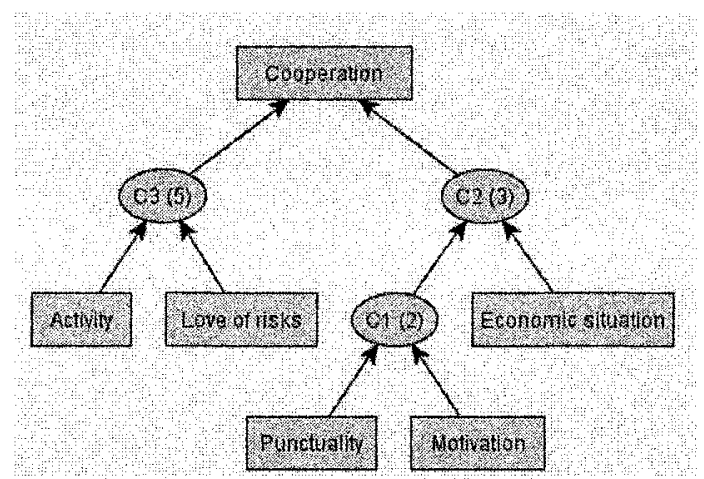

Figure 4-Hierarchical structure obtained by HINT showing that Activity and Love of risks more directly influence Cooperation than Punctuality and Motivation

\section{CONCLUSIONS AND FURTHER RESEARCH}

In this paper we proposed a general multi-attribute decision-making model for VO creation. We first formalized VO selection as a multi-criteria portfolio-selection problem and secondly suggested an attribute hierarchy for selection criteria. The approach was illustrated through an example of an operating VBE, the Virtuelle Fabrik AG, Switzerland.

The multi-criteria decision-analysis methodology seems to work as a good approach for supporting VO selection. Its advantages are strongly developed theory, transparency of decision criteria, an ability to use models for various analyses and simulations, such as what-if analysis, and for improved communication and documentation between decision-makers themselves as well as between decisionmakers and other interested parties. Usually, decision-making models are applicable as software tools, albeit computational challenges may arise if the set of alternatives becomes very large. In addition, the existence of multiple decision-makers makes the problem very difficult (e.g. Arrow, 1951).

This paper suggests several avenues for future research. First, performance measurement, especially for network-related attributes, is called for. Second, suitable methods for attribute-weight elicitation are needed. For instance, we have identified the need to cope with qualitative variables and combine both quantitative and qualitative criteria. Third, the practical value of multi-attribute decision-support methodology to VO creation should be evaluated through an extensive real-life case study, which could additionally lead to tool development. 


\section{ACKNOWLEDGEMENTS}

This work was supported by the 6FP Integrated Project ECOLEAD, the Slovenian Ministry of Higher Education, Science and Technology, and the National Technology Agency of Finland (Tekes) and the National Workplace Development Programme (Tykes) through the COBTEC project.

\footnotetext{
${ }^{\mathrm{i}}$ This term was suggested by Mario Martínez in an ECOLEAD project meeting in Rome, January 2005.
}

\section{REFERENCES}

1. Arrow K. Social Choice and Individual Values, PhD Thesis, Columbia University, 1951.

2. Bohanec M. "Decision Support". In Data Mining and Decision Support: Integration and Collaboration, Mladenić D, Lavraæ N, Bohanec M, Moyle S, ed. Kluwer Academic Publishers, 2003.

3. Bohanec M, Rajkovið V. Multi-Attribute Decision Modeling: Industrial Applications of DEX. Informatica 23(2), 487-491, 1999.

4. Bohanec M, Zupan B. A Function-Decomposition Method for Development of Hierarchical MultiAttribute Decision Models. Decision Support Systems 2004; 36: 215-33.

5. Camarinha-Matos LM, Afsarmanesh $\mathrm{H}$. Elements of a base VE infrastructure. Computers in Industry $2003 ; 51(2): 139-63$.

6. Lin $\mathrm{C}-\mathrm{W}$, Chen $\mathrm{H}-\mathrm{Y}$. A fuzzy strategic alliance selection framework for supply chain partnering under limited evaluation resources. Computers in Industry $2004 ; 55(2): 159-79$.

7. Demsar J, Zupan B. Orange: From Experimental Machine Learning to Interactive Data Mining. White paper, Faculty of Computer and Information Science, University of Ljubljana, Slovenia, 2004. http://www.ailab.si/orange

8. Edwards W, Barron FH. SMARTS and SMARTER: Improved Simple Methods for Multiattribute Utility Measurement. Organizational Behavior and Human Decision Processes 1994; 60: 306-25.

9. Katzy BR, Dissel M. A Toolset for Building the Virtual Enterprise. Journal of Intelligent Manufacturing 2001; 12:121-31.

10. Keeney RL, Raiffa H. Decisions with Multiple Objectives: Preferences and Value Tradeoffs, John Wiley \& Sons, 1976.

11. Liesiö J, Mild P, Salo A. Preference Programming for Robust Portfolio Modeling and Project Selection, working paper, Systems Analysis Laboratory, Helsinki University of Technology, 2005. http://www.sal.hut.fi/Publications/pdf-files/mlie05.pdf

12. Saaty TL. Multicriteria Decision Making: The Analytic Hierarchy Process. RWS Publications, 1993.

13. Triantaphyllou E. Multi-Criteria Decision Making Methods: A Comparative Study. Kluwer Academic Publishers, 2000.

14. Zupan B, Bohanec M, Demsar J, Bratko I. Learning by discovering concept hierarchies. Artificial Intelligence 1999; 109: 211-42. 